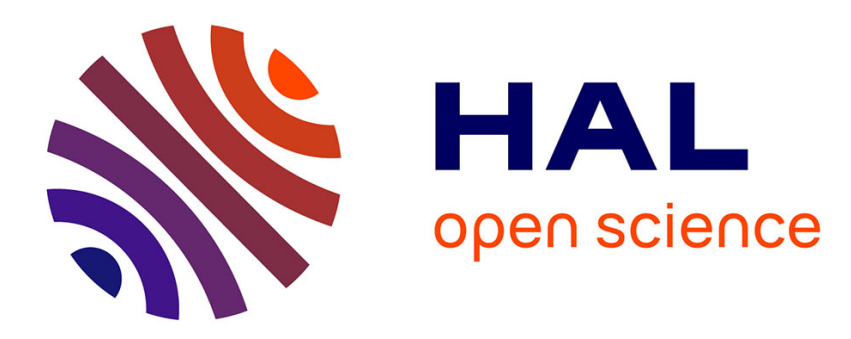

\title{
Le roi de France est déjà chauve: remarques sur l'antériorité temporelle du présupposé
}

\author{
Sandrine Deloor
}

\section{To cite this version:}

Sandrine Deloor. Le roi de France est déjà chauve : remarques sur l'antériorité temporelle du présupposé. 2012. hal-00685770

\section{HAL Id: hal-00685770 \\ https://hal.science/hal-00685770}

Preprint submitted on 6 Apr 2012

HAL is a multi-disciplinary open access archive for the deposit and dissemination of scientific research documents, whether they are published or not. The documents may come from teaching and research institutions in France or abroad, or from public or private research centers.
L'archive ouverte pluridisciplinaire HAL, est destinée au dépôt et à la diffusion de documents scientifiques de niveau recherche, publiés ou non, émanant des établissements d'enseignement et de recherche français ou étrangers, des laboratoires publics ou privés. 
Version pre-print. A paraître dans Langages.

Le roi de France est déjà chauve : remarques sur l'antériorité temporelle du présupposé

\author{
Sandrine Deloor \\ UMR 7187 LDI, Université de Cergy-Pontoise
}

\title{
Introduction
}

La majorité des auteurs qui se sont intéressés à l'adverbe déjà (ou à l'un de ses équivalents dans une autre langue) ont eu recours à la notion de présupposition pour décrire son fonctionnement sémantique. Cependant, les modèles proposés sont très différents les uns des autres : pour certains, déjà présuppose une phase antérieure au moment de référence de l'énoncé tandis que pour d'autres, c'est à une phase postérieure (présentée comme une simple possibilité dans certains modèles) que renvoie cet adverbe. Dans la mesure où les auteurs s'appuient sur les mêmes tests pour identifier les présupposés véhiculés par déjà, on peut tout d'abord s'étonner qu'une telle diversité d'approches soit possible. Mais il y a plus grave encore : certaines des hypothèses présentées mettent au jour d'importantes divergences dans l'interprétation même du concept de présupposé. Dans certaines descriptions, par exemple, les approches vériconditionnelle et sémantico-pragmatique de la présupposition se révèlent incompatibles, soit parce qu'elles donnent lieu à des interprétations très différentes d'une même hypothèse, soit parce que l'une d'elle ne peut pas être appliquée. Par ailleurs, les auteurs ne prennent pas toujours en compte l'antériorité temporelle du présupposé, ce qui, dans certains modèles, revient à nier sa spécificité.

Notre objectif, dans cet article, sera d'une part d'exposer et d'analyser les divergences entre les différentes descriptions de déjà et, d'autre part, de mettre en évidence la valeur explicative de l'antériorité présuppositionnelle ${ }^{1}$.

\section{Des approches différentes}

Dans cette première partie de notre étude, nous présentons trois descriptions sémantiques de déjà ou équivalent, basées sur la notion de présupposition ${ }^{2}$. Dans les trois cas, les auteurs s'appuient sur le critère de négation : est considéré comme présupposé ce qui est commun à Déjà $P(t)$ et $N e$ pas encore $P(t)$ ( $\mathrm{t}$ renvoyant au moment de référence de $P$ ).

\subsection{Modèle 1 : une présupposition concernant le passé}

Pour les défenseurs du modèle 1 (Abraham, 1980 ; Garrido Medina, 1992), l'adverbe déjà marque un changement par rapport à une phase antérieure présupposée. Cette hypothèse est exposée comme suit par Garrido Medina (1992) :

(5) María vive aquí ya.

"María lives here already."

(6) María no vive aquí todavía.

"María does not live here yet."

Both (5) and (6) entail (7), while they respectively entail (8) and (9), so that they have (7) as a presupposition :

(7) María no vivía aquí antes.

[...] "Mary didn't live here before."

(8) María vive aquí.

1 Nous n'aborderons ici que les énoncés où l'adverbe déjà est associé à l'aspect imperfectif. Pour une étude des relations entre déjà et l'aspect perfectif, voir Deloor (2010).

2 L'adverbe déjà et ses équivalents ont fait l'objet d'une littérature abondante dont nous ne donnons ici qu'un bref aperçu. Pour une bibliographie plus complète, voir Deloor (2006). 
Version pre-print. A paraître dans Langages.

"Mary lives here."

(9) María no vive aquí.

[...] "Mary doesn't live here." (Garrido Medina, $1992: 359-360$ )

Nous proposons ci-dessous un tableau résumant cette approche :

MODELE 1

\begin{tabular}{|l|l|l|}
\hline & Présupposé & Posé \\
\hline Déjà $P(t)$ & $\exists \mathrm{t}^{\prime}: \mathrm{t}^{\prime}<\mathrm{t} \& \sim \mathrm{P}\left(\mathrm{t}^{\prime}\right)^{3}$ & $\mathrm{P}\left(\mathrm{t}^{4}\right.$ \\
\hline
\end{tabular}

\subsection{Modèle 2 : une présupposition concernant une possibilité future}

Le modèle 2 a été défendu par Doherty (1973) et Martin (1983b). Selon ces auteurs, déjà véhicule une présupposition concernant le passé et une présupposition concernant une possibilité future :

Dire que Pierre était déjà là à $8 \mathrm{~h}$, c'est présupposer qu'il est au moins possible qu'il n'ait pas été là avant $8 \mathrm{~h}$. ( D’où l'inacceptabilité de *Il est déjà jeune, *Il est déjà petit, *Il est déjà trop tôt pour le dire ...). Pierre n'était pas encore là à $8 h$ suppose qu'il n'était pas là avant $8 \mathrm{~h}$ (absence qui implique pour le moins sa propre possibilité). Et les deux phrases laissent entendre que la présence de Pierre est au moins possible après $8 \mathrm{~h}$. (D'où l'impossibilité de dire, dans un éloge funèbre - exemple de C. Muller : *Il a déjà fait du bien dans sa vie.) (Martin, 1983b : 51)

On a :

MODELE 2

\begin{tabular}{|l|l|l|}
\hline & Présupposé & Posé \\
\hline Déjà $P(t)$ & $\begin{array}{l}\exists \mathrm{t}^{\prime}: \mathrm{t}^{\prime}<\mathrm{t} \& \sim \mathrm{P}\left(\mathrm{t}^{\prime}\right) \\
\exists \mathrm{t}^{\prime},: \mathrm{t}^{\prime} \mathrm{t}^{\prime} \& \diamond \mathrm{P}\left(\mathrm{t}^{\prime},\right)^{5}\end{array}$ & $\mathrm{P}(\mathrm{t})$ \\
\hline
\end{tabular}

\subsection{Modèle 3 : une présupposition concernant le futur}

Pour les défenseurs du modèle 3 (Horn, 1970 ; Muller, 1975 ; König, 1977), c'est sur une phase postérieure au moment de référence de l'énoncé que porte la présupposition activée par déjà :

It is already raining. / It isn't raining yet.

$\mathrm{P}:$ It will be raining sometime later.

A : It is raining now. / It isn't raining now. (Horn, $1970: 321)^{6}$

On a :

MODELE 3

\begin{tabular}{|l|l|l|}
\hline & Présupposé & Posé \\
\hline Déjà $P(t)$ & $\exists \mathrm{t}^{\prime}: \mathrm{t}^{\prime}>\mathrm{t} \& \mathrm{P}\left(\mathrm{t}^{ }\right)^{7}$ & $\mathrm{P}(\mathrm{t})$ \\
\hline
\end{tabular}

\section{Des hypothèses ambiguës}

Deux des modèles que nous venons de présenter font de déjà un adverbe orienté vers le futur. Après avoir rappelé les différences entre les approches vériconditionnelle et sémantico-pragmatique de la présupposition, nous montrerons que cette hypothèse soulève d'importants problèmes d'interprétation.

Il existe t' tel que t' est strictement antérieur à $\mathrm{t}$ et non-P est vrai en t'.

P est vrai en $t$.

Il existe $\mathrm{t}$ "' tel que $\mathrm{t}$ "' est strictement postérieur à $\mathrm{t}$ et il est possible que $\mathrm{P}$ soit vrai en $\mathrm{t}$ "'

Dans cette citation, «P $\mathrm{P}$ est mis pour «Presupposition» et « $\mathrm{A} »$ pour « Assertion».

Il existe $\mathrm{t}$ ' tel que $\mathrm{t}$ ' est strictement postérieur à $\mathrm{t}$ et $\mathrm{P}$ est vrai en $\mathrm{t}$ '. 


\subsection{Approche vériconditionnelle vs. Approche sémantico-pragmatique}

Dans l'approche vériconditionnelle issue de Frege (1892) et Strawson (1950), le présupposé est vu comme une condition d'évaluation : dire que A présuppose $\mathrm{B}$ équivaut à dire que $\mathrm{B}$ doit être vrai pour que A ait une valeur de vérité. On remarquera que cette définition n'accorde pas de statut particulier au posé : la présupposition n'est pas vue comme une relation entre une proposition présupposée et une proposition posée mais comme une relation entre une proposition présupposée et l'énoncé qui la présuppose. Dire que l'énoncé Pierre continue à fumer présuppose que < Pierre fumait avant > équivaut à dire que la proposition < Pierre fumait avant > doit être vraie pour que l'on puisse attribuer une valeur de vérité à l'énoncé Pierre continue à fumer (et non pour que l'on puisse attribuer une valeur de vérité à la proposition posée $\langle\text { Pierre fume actuellement }>)^{8}$.

Cette approche «conditionnaliste" suppose une temporalité de l'évaluation: la proposition présupposée doit être évaluée avant l'énoncé puisque ce n'est que si elle est vraie que l'évaluation de l'énoncé est possible.

Nous opposerons à cette conception l'approche sémantico-pragmatique de Ducrot $(1972,1981)$ et Anscombre (1990b). Pour ces auteurs, le présupposé n'est pas une condition d'emploi mais un élément de contenu ${ }^{9}$ et il est indissociable du posé. Dire que A présuppose $\mathrm{B}$ et pose $\mathrm{C}$ équivaut à dire qu'en énonçant $\mathrm{A}$, le locuteur fait comme si $\mathrm{ON}^{10}$ savait que $\mathrm{B}$ avant l'énonciation et que c'est dans ce cadre qu'il asserte que C. Ainsi, dire que l'énoncé Pierre continue à fumer présuppose que < Pierre fumait avant > et pose que < Pierre fume actuellement > équivaut à dire qu'en énonçant Pierre continue à fumer, le locuteur fait comme si ON savait, avant l'énonciation, que < Pierre fumait avant $>$ et que c'est dans cadre qu'il asserte que $<$ Pierre fume actuellement $>$.

Comme l'approche vériconditionnelle, l'approche sémantico-pragmatique met l'accent sur l'antériorité temporelle du présupposé : le présupposé est présenté par le locuteur comme un savoir antérieur au moment de l'énonciation et comme le cadre dans lequel s'inscrit le posé.

Lorsque l'on étudie les exemples paradigmatiques de constructions présuppositionnelles, ces deux approches de la présupposition ne semblent pas incompatibles : on a l'impression qu'il s'agit simplement de deux points de vue différents sur un même phénomène (cf. Ducrot, 1972: 25-26). Nous montrons ci-dessous qu'il en va tout autrement lorsque l'on s'intéresse à un exemple non paradigmatique tel que déjà.

\subsection{Possibilité future et mise en perspective}

\footnotetext{
${ }^{8}$ Cette opposition entre «proposition» et «énoncé » est extrêmement importante car elle permet d'établir une distinction entre ce qui relève de la langue (l'énoncé) et ce qui relève de la métalangue (la proposition). Elle est difficile à percevoir dans l'exemple Le roi de France est chauve où, comme le souligne Kleiber dans ce numéro, « le présupposé est dans le posé ». Pour ce type d'exemple, seule l'approche de Russell permet d'éviter que la présupposition ne contamine la métalangue : l'existence du roi de France n'est pas présupposée dans la formule $<$ il existe un seul $\mathrm{x}$ tel que $x$ est roi de France et $x$ est chauve $>$. Dans l'approche de Frege, en revanche, la présupposition est dans ce cas un phénomène aussi bien linguistique que logique : la proposition < le roi de France est chauve > présuppose, comme l'énoncé Le roi de France est chauve, qu'< il existe un roi de France $>$. Pour l'exemple Pierre continue à fumer, le problème se pose différemment : dans une perspective strictement logique, il est inutile de s'embarrasser d'un prédicat tel que < continuer à fumer > puisque le contenu ainsi exprimé en langue peut être décomposé en deux propositions indépendantes (il n'est pas nécessaire de pouvoir évaluer $<$ Pierre fumait avant $>$ pour pouvoir évaluer $<$ Pierre fume actuellement $>$ ) ; dans ce type d'exemple, c'est uniquement pour décrire l'énoncé de la langue (dans une perspective linguistique, donc) que la notion de présupposition est pertinente.

9 En proposant cette approche, Ducrot et Anscombre s'opposent non seulement à l'approche vériconditionnelle issue de Frege et Strawson (dans laquelle le présupposé est vu comme une condition d'emploi logique) mais aussi à des auteurs comme Fillmore (1970) ou Stalnaker (1974) pour qui le présupposé est une condition d'emploi pragmatique.

${ }^{10} \mathrm{Ce} \mathrm{ON}$ renvoie à une communauté discursive à laquelle le locuteur dit appartenir (cf. la notion de «ON-locuteur» de Anscombre, 2005).
} 
Pour Löbner $(1989,1999)$, les conditions de vérité de Schon $P(t)$ (équivalent allemand de Déjà $P(t)$ ) et de Noch nicht $P(t)$ (équivalent allemand de Ne pas encore $P(t)$ ) peuvent être formulées comme suit (soit $t_{e}$, le moment de référence de $P$ ) :

Truth conditions for type-S schon $\left(t_{e}, P\right)$ and noch nicht $\left(t_{e}, P\right)$ :

a- Both schon $\left(t_{e}, P\right)$ and noch nicht $\left(t_{e}, P\right)$ trigger the presupposition that there is a phase of not $-\mathrm{P}$ starting before $\mathrm{t}_{\mathrm{e}}$ and that up to $t_{\mathrm{e}}$ at most one change between not-P and $\mathrm{P}$ has occurred.

b- Schon $\left(t_{e}, P\right)$ is true, and noch nicht $\left(t_{e}, P\right)$ is false, iff the presupposition in (a) is fulfilled and $P\left(t_{e}\right)$ is true.

c- Schon $\left(t_{e}, P\right)$ is false, and noch nicht $\left(t_{e}, P\right)$ is true, iff the presupposition in (a) is fulfilled and $P\left(t_{e}\right)$ is false. (Löbner, 1999 : 53-54)

Si la proposition de Löbner présente des similitudes avec le modèle 1, une différence de taille est cependant à signaler : pour Löbner, le présupposé véhiculé par Déjà $P(t)$ ne concerne pas seulement l'existence d'une phase antérieure à $\mathrm{t}$ où $\mathrm{P}$ n'était pas vrai. En plus de cette information, Déjà $P(t)$ présuppose qu'il y a eu au plus un changement entre non-P et $\mathrm{P}$ avant $\mathrm{t}$ ( up to $\mathrm{t}_{\mathrm{e}}$ at most one change between not-P and $\mathrm{P}$ has occurred »). C'est cet ajout qui distingue la proposition de Löbner du modèle 1 .

Comment comprendre cette formulation? Même si l'analyse de Löbner relève d'une approche vériconditionnelle de la présupposition ${ }^{11}$, nous pensons que ce n'est que lorsque l'on adopte un point de vue sémantico-pragmatique qu'elle prend tout son sens.

Dans un cadre vériconditionnel, dire que Déjà $P(t)$ présuppose qu'il y a eu au plus un changement entre non-P et $\mathrm{P}$ avant $\mathrm{t}$ équivaut à dire que, pour que l'on puisse attribuer une valeur de vérité à Déjà $P(t)$, il faut qu'il y ait effectivement eu un changement au plus (autrement dit 0 ou 1 changement) entre non-P et $\mathrm{P}$ avant $\mathrm{t}$. On voit mal en quoi cette formulation diffère de l'hypothèse d'une présupposition concernant le passé.

Lorsque 1'on adopte un cadre sémantico-pragmatique, en revanche, on aboutit à une interprétation tout à fait différente : dans ce cas, dire que Déjà $P(t)$ présuppose qu'il y a eu au plus un changement entre non-P et $\mathrm{P}$ avant $\mathrm{t}$ équivaut à dire qu'en énonçant Déjà $P(t)$, le locuteur fait comme si $\mathrm{ON}$ savait, avant l'énonciation, qu'il y avoir ${ }^{12}$ un changement au plus entre non-P et $\mathrm{P}$ avant $\mathrm{t}$. Autrement dit, ON savait, avant l'énonciation, qu'un changement entre non-P et $\mathrm{P}$ être-susceptible de se produire avant $t$. C'est dans ce cadre que s'inscrit l'assertion : la situation en t est envisagée non seulement par rapport à une phase antérieure où $\mathrm{P}$ n'était pas vrai mais aussi par rapport à la possibilité d'un changement entre non-P et $\mathrm{P}$ avant $\mathrm{t}$.

Si notre interprétation est correcte, l'analyse de Löbner ne se réduit pas à l'hypothèse d'une présupposition concernant le passé. Pour cet auteur, il s'agit également de mettre l'accent sur la perspective introduite par l'adverbe déjà. Cette idée apparaît clairement dans la citation suivante :

In agreement with Vandeweghe $(1979,1983)$ I prefer to talk of "perspectives" instead of "presuppositions". The particles schon and noch put the state $p$ under a certain specific perspective. Schon $\left(t_{e}, P\right)$ introduces the perspective that after a phase of not $-p$ a positive phase sets in and, under this perspective, states that $p$ is the case at the reference time. (Löbner,1989 : 174-175)

L'hésitation terminologique de Löbner est significative. Comme nous l'avons vu, seule une approche sémantico-pragmatique permet de donner tout son sens à l'hypothèse qu'il présente. D'une façon plus générale, l'idée de mise en perspective qui est au centre de cette hypothèse est difficilement conciliable avec les principes de base de la sémantique vériconditionnelle :

\footnotetext{
${ }^{11}$ Cette position apparaît clairement à la fin de son article de 1999 où il indique qu'il s'appuie sur « the Fregean concept of presupposition » qu'il définit comme suit : «the conditions common to the cases of being true and being false » (Löbner, 1999 : 105)

${ }^{12}$ A plusieurs reprises dans cette section et dans les suivantes, nous avons recours à l'infinitif pour éviter certains problèmes liés à la concordance des temps en français. Ces problèmes seront expliqués en 3.2.
} 
Version pre-print. A paraître dans Langages.

In a sense which will be made more precise later, the approach taken here is operational rather than truth-conditional. The posing of a perspective is something which does not fit into the usual setting of model-theoretic semantics with a direct correspondence between linguistic forms and a model-structure. (Löbner, 1989 : 175)

Comme l'hypothèse de Löbner (1989, 1999), le modèle 2 peut faire l'objet de deux interprétations différentes selon que l'on adopte une approche vériconditionnelle ou sémantico-pragmatique de la présupposition. Pour ses défenseurs, nous l'avons vu, Déjà $P(t)$ présuppose (i) que $\mathrm{P}$ n'était pas vrai à un moment antérieur à $\mathrm{t}$ et (ii) qu'il est possible que $\mathrm{P}$ soit vrai à un moment postérieur à $\mathrm{t}$. Examinons l'hypothèse (ii) :

(ii - a) Interprétation vériconditionnelle : Il faut qu'il soit possible que $\mathrm{P}$ soit vrai à un moment postérieur à t pour que Déjà $P(t)$ ait une valeur de vérité.

(ii - b) Interprétation sémantico-pragmatique : En énonçant Déjà $P(t)$, le locuteur fait comme si ON savait, avant l'énonciation, qu'il être-possible que $\mathrm{P}$ soit vrai à un moment postérieur à t. Dans ce cadre, il asserte que $\mathrm{P}$ est vrai en t.

On conviendra que ces deux interprétations ne sont en aucun cas équivalentes. En (ii - a), le présupposé est assimilé à une contrainte combinatoire et contextuelle : il n'intervient dans la description sémantique que pour expliquer l'inacceptabilité de déjà dans certains énoncés (\# Il est déjà jeune) et dans certaines situations de discours (\# Il a déjà fait du bien dans sa vie, à propos d'un défunt). En (ii - b), en revanche, le présupposé est un contenu à part entière : on fait l'hypothèse que tout énoncé comportant déjà met en perspective la situation en $t$ (contenu posé) par rapport à une situation postérieure possible, envisagée avant l'énonciation (contenu présupposé) ${ }^{13}$.

On remarquera que la seconde interprétation rapproche le modèle 2 de l'hypothèse de Löbner. En considérant que déjà véhicule (i) un présupposé concernant une phase antérieure non-P et (ii) un présupposé concernant une possible phase postérieure $\mathrm{P}$, ses défenseurs mettent l'accent, comme Löbner, sur la perspective introduite par cet adverbe : la situation en $\mathrm{t}$ (posé) est envisagée par rapport à la possibilité d'un changement entre non-P et $\mathrm{P}$ (présupposé). Notons cependant que, comme pour l'hypothèse de Löbner, c'est seulement en adoptant une approche sémanticopragmatique de la présupposition que l'on aboutit à cette interprétation du modèle 2. L'approche vériconditionnelle engendre quant à elle une interprétation tout à fait différente.

\subsection{Le problème de la présupposition concernant le futur}

Pour les défenseurs du modèle 3 , Déjà $P(t)$ présuppose que $\mathrm{P}$ sera vrai à un moment postérieur à $\mathrm{t}$. Dans un cadre vériconditionnel, cela signifie que, pour que Déjà $P(t)$ ait une valeur de vérité, il faut que $\mathrm{P}$ soit effectivement vrai à un moment postérieur à t. Pour certains auteurs, cette interprétation du modèle 3 soulève un important problème : si t renvoie au moment de l'énonciation, alors la proposition $<\mathrm{P}$ est vraie à un moment postérieur à $\mathrm{t}>\mathrm{n}$ 'est pas évaluable. Cette objection est formulée comme suit par Löbner (1989) ${ }^{14}$ :

Some authors adopted the view that the actual or a possible course of events after the time $t_{\mathrm{e}}$ is part of the truthconditions of schon $\left(t_{e}, p\right)$ and noch $\left(t_{e}, p\right)$. This question, however, can be handled in a radically minimalistic manner : The truth or falsity of schon $\left(t_{e}, p\right)$ and noch $\left(t_{e}, p\right)$ does not impose any contraints at all on the time after $\mathrm{t}_{\mathrm{e}}[\ldots]$ Horn (1970) (as well as König, 1977) postulated that the truth of schon $\left(t_{e}, p\right)$ implies that the state $p$ extends beyond $t_{e}$. If this were in fact a condition for the truth of that sentence this would mean that one can truely utter schon $\left(t_{e}, p\right)$ only if one knows how things go on with respect to $p$ after the time $t_{\mathrm{e}}$. This is not the case. (Löbner, $1989: 176$ )

\footnotetext{
${ }^{13}$ Notons que (ii - b) implique (ii - a) : dire que l'un des contenus véhiculés par déjà concerne la possibilité que $\mathrm{P}$ soit vrai après $\mathrm{t}$ implique que l'emploi de déjà n'est acceptable que si $\mathrm{P}$ est effectivement possible après $\mathrm{t}$. L'approche sémantico-pragmatique permet donc de faire les mêmes prédictions que l'approche vériconditionnelle même si la notion de présupposition y est utilisée pour décrire le sens des énoncés et non les conditions pour que les énoncés aient du sens.

${ }^{14}$ On trouve le même type d'objection chez Garrido Medina (1991 : 50) et Girón Alconchel (1991 : 24).
} 
On conviendra que Löbner est particulièrement injuste avec Horn et König : contrairement à ce qu'il laisse entendre, il semble évident que ces auteurs n'ont jamais voulu dire que les locuteurs étaient des devins! Cela étant - et aussi triviale qu'elle puisse paraître - l'objection de Löbner est difficile à contester dans un cadre strictement vériconditionnel. Si des solutions générales ont été proposées pour résoudre le problème de l'inévaluabilité de certains énoncés, elles semblent cependant peu compatibles avec la nature particulière du présupposé. Horn (1997) propose par exemple d'établir une distinction entre les « conditions de vérité » d'un énoncé et ses « conditions de vérification»:

This approach extends naturally to other familiar cases of sentences that must be either true or false but cannot be felicitously uttered (in a given context), including future contingent statements (e.g. Aristotle's There will be a seabattle tomorrow), past "unknowables" (e.g. There was an odd number of blades of grass in Harvard Yard at the dawn of Commencement Day, 1903, from Quine 1981: 91) or unverifiable and unfalsifiable claims about the present (e.g. the medievals' The number of stars is even). A theory which severs questions of truth conditions from those of verification can account for such cases without sacrificing either classical bivalence or our intuitions of conditions on (un)assertibility (Horn, 1997 : 304).

Si cette solution permet de rendre compte des énoncés inévaluables, résout-elle pour autant le problème soulevé par Löbner? N'est-il pas problématique que, dans certains cas, la condition pour qu'un énoncé soit évaluable (le présupposé) soit elle-même inévaluable?

Cette question est d'autant plus prégnante que l'objection de Löbner peut être étendue à d'autres constructions présuppositionnelles. L'admettre conduirait par exemple à mettre en question les descriptions traditionnelles du verbe savoir et des constructions clivées :

Pierre sait que Paul viendra.

$>$ Le présupposé habituellement identifié ici $(<$ Paul viendra $>$ ) n'est pas évaluable au moment de l'énonciation.

C'est Marie qui embrassera Jean.

$>$ Le présupposé habituellement identifié ici (< Quelqu'un embrassera Jean $>$ ) n'est pas évaluable au moment de l'énonciation.

Il n'est évidemment pas question de trancher ici un tel débat. Si nous avons choisi de commenter la citation de Löbner (1989), c'est aussi et surtout parce qu'elle repose sur une interprétation du modèle 3 qui nous semble discutable. Pour Löbner, dire que Déjà $P(t)$ présuppose que $\mathrm{P}$ est vrai à un moment postérieur à $\mathrm{t}$ revient à dire que Déjà $P(t)$ indique que $\mathrm{P}$ continue après $\mathrm{t}$ ( « the state $p$ extends beyond $\left.t_{\mathrm{e}} »\right)$. On remarquera qu'en proposant cette interprétation du modèle 3 , Löbner ne tient pas compte de l'antériorité temporelle du présupposé. Pour savoir si $\mathrm{P}$ « continue » après $\mathrm{t}$, il faut savoir si $\mathrm{P}$ est vrai en $\mathrm{t}$. Or, dans le modèle 3 , la proposition $<\mathrm{P}$ est vrai en $\mathrm{t}\rangle$ correspond au posé, c'est-à-dire que l'on considère que cette proposition n'est prise en compte qu'après l'évaluation du présupposé (approche vériconditionnelle) ou dans le cadre du présupposé (approche sémantico-pragmatique). Même si le modèle 3 peut parfois être interprété en termes de continuation (nous le verrons en 3.2.), ce n'est d'une part pas toujours le cas et d'autre part il ne peut s'agir que d'une conséquence de son application : l'idée de continuation ne peut en aucun cas apparaître dans la formulation du présupposé car cela impliquerait que le présupposé est subordonné au posé ; or, tant dans l'approche vériconditionnelle que dans l'approche sémanticopragmatique, c'est l'inverse qui est postulé.

\section{L'épreuve des faits}

Une fois construites, les théories doivent être confrontées aux faits dont elles cherchaient à rendre compte. Or, en sémantique, il est rare que les faits en question soient formulés de façon explicite. Les auteurs construisent des modèles, montrent que, grâce à eux, ils sont en mesure de prédire certains phénomènes (des inacceptabilités le plus souvent) mais présentent rarement de façon systématique l'ensemble des faits qu'ils cherchaient à expliquer. La confrontation que nous proposons dans cette dernière partie est donc quelque peu paradoxale: nous confrontons les interprétations que nous avons données des différents modèles (interprétations avec lesquelles les 
Version pre-print. A paraître dans Langages.

auteurs ne seraient peut-être pas d'accord) à des observations qui n'apparaissent pas dans les études citées (et que certains auteurs pourraient donc rejeter).

\subsection{Une discontinuité par rapport à une phase antérieure}

Soit le dialogue suivant, proposé par Mittwoch (1993 :74)

(1) A- I’ve applied for American citizenship.

B- Is your husband also applying?

A- He is already American, for he was born in America.

Pour les défenseurs des modèles 1 et 2, l'énoncé He is already American présuppose l'existence d'une phase antérieure au moment de référence où [il être américain] n'était pas vrai. Etant donné que l'énoncé He was born in America implique que le sujet a toujours été américain, la réplique de A devrait être contradictoire. Ce n'est pas le cas. Cet exemple vient donc contredire l'hypothèse selon laquelle l'adverbe déjà et ses équivalents activent une présupposition concernant le passé.

L'objection de Mittwoch a fait l'objet d'un vif débat dans la littérature. Pour van der Auwera (1993), par exemple, il est faux de dire que l'énoncé He was born in America implique que le sujet a toujours été américain :

[...] the situation of the husband being American is still preceded by a situation for which it is not true that he was American. Before his birth he didn't exist yet, hence he wasn't American either. But this is communicatively fully irrelevant. (van der Auwera, $1993: 622$ )

Michaelis (1996) rejette l'argumentation de van der Auwera. Selon elle, un énoncé portant sur un être qui n'est pas encore né est inévaluable car la présupposition d'existence qu'il véhicule n'est pas satisfaite :

(5) I have been American for 31 years. Before that, I wasn't American.

Sentence (5) [...] invoques the problem of satisfaction of the existential presupposition invoked by the pronominal subject $I$. If the speaker did not exist prior to 1964, then the existential presupposition is not satisfied, and, if one suscribes to a theory in which there are truth gaps, an assertion like In 1963, I wasn't an American cannot be evaluated as true or false. (Michaelis, $1996: 481$ )

Ce débat sur la nationalité de personnes qui n'existent pas peut laisser perplexe... Il relève en fait d'une conception référentialiste de la langue et repose sur deux hypothèses implicites : (i) les énoncés décrivent la réalité et (ii) décrire le sens d'un énoncé, c'est décrire la réalité à laquelle il réfère. Nous adoptons pour notre part une conception différente, inspirée des travaux de J.C. Anscombre et O. Ducrot. Pour nous, le sens d'un énoncé n'est pas une description mais une représentation du réel. Les différentes implications logiques ou empiriques d'un énoncé ne font pas nécessairement partie de son sens car elles ne sont pas nécessairement incluses dans la représentation mise en place par cet énoncé. Dans He is already American, for he was born in America, aucune phase antérieure t' telle que [le mari de A ne pas être américain en t'] n'est évoquée et c'est pour nous une raison suffisante pour remettre en cause l'hypothèse selon laquelle already active une présupposition concernant le passé. La question de savoir si les gens ont une nationalité avant leur naissance ne nous semble pas être une question linguistique et nous considérons qu'elle n'est pas pertinente pour décrire cet énoncé.

\subsection{Expectative et continuation}

Soient les énoncés suivants :

(2) Paul veut déjà être ministre.

(3) En 2007, Paul voulait déjà être ministre. 
Selon nous, $(2)$ véhicule trois contenus : $(2 a)<$ Actuellement Paul veut être ministre $>,(2 b)<$ On s'attendait à ce que Paul ressente un jour l'envie d'être ministre > et $(2 \mathrm{c})<$ On ne s'attendait pas à ce que Paul ressente si tôt l'envie d'être ministre >. En d'autres termes, nous considérons que (2) indique qu'une expectative (contenu 2b) s'est réalisée (contenu 2a) plus tôt que prévu (contenu 2c). En (3), nous distinguons les deux contenus suivants : (3a) < En 2007, Paul voulait être ministre > et (3b) < Après 2007, Paul voulait être ministre >. En d'autres termes, nous considérons que (3) indique que la situation en vigueur en 2007 (contenu 3a) a continué après 2007 (contenu 3b).

L'inacceptabilité des enchaînements suivants corrobore notre description de (2) :

(4) \# Paul n'a jamais fait de politique et pourtant il veut déjà être ministre.

(5) \# Paul veut déjà être ministre? C'est pas trop tôt!

En (4), il y a une opposition entre le contenu (2b) (< On s'attendait à ce que Paul ressente un jour l'envie d'être ministre >) et l'énoncé Paul n'a jamais fait de politique, qui indique que rien dans le comportement de Paul ne laissait supposer qu'il aurait envie un jour d'être ministre. En (5), le locuteur se contredit en soutenant d'une part qu'il ne s'attendait pas à ce que Paul ressente si tôt l'envie d'être ministre (contenu 2c) et d'autre part que cela faisait longtemps qu'il attendait que cela arrive (C'est pas trop tôt).

On remarquera que ces inacceptabilités disparaissent lorsque l'on supprime l'adverbe déjà :

(6) Paul ne fait pas de politique et pourtant il veut être ministre.

(7) Paul veut être ministre? C'est pas trop tôt!

On en déduit que c'est l'adverbe déjà qui est responsable des contenus (2b) et (2c).

Pour se convaincre de la présence du contenu (3b) (< Après 2007, Paul voulait être ministre >) en (3) (En 2007, Paul voulait déjà être ministre), on comparera les exemples suivants :

(8) \# En 2007, Paul voulait déjà être ministre mais il a changé d'avis quand il a vu le gouvernement à l'œuvre.

(9) En 2007, Paul voulait être ministre mais il a changé d'avis quand il a vu le gouvernement à l'œuvre.

En (8), le contenu (3b) entre en contradiction avec le changement exprimé dans la seconde proposition. En (9), la suppression de déjà rend l'enchaînement acceptable.

Dans ce qui suit, nous nous proposons de montrer que le modèle 3 permet de rendre compte de l'ensemble des faits qui viennent d'être observés. Précisons que, dans la mesure où ce modèle est incompatible avec l'approche vériconditionnelle de la présupposition (cf. 2.3), c'est uniquement à son interprétation sémantico-pragmatique que nous nous intéressons.

D'un point de vue sémantico-pragmatique, dire que Déjà $P(t)$ présuppose que $\mathrm{P}$ est vrai à un

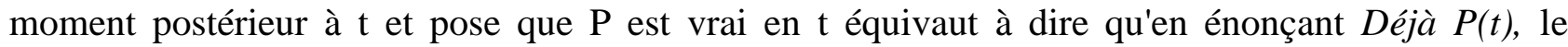
locuteur fait comme si on savait, avant l'énonciation, que $\mathrm{P}$ être-vrai à un moment postérieur à t et que c'est dans ce cadre qu'il asserte que $\mathrm{P}$ est vrai en $\mathrm{t}$. Avant d'appliquer cette hypothèse, il convient de remarquer qu'elle repose sur une distinction entre quatre moments différents : outre le moment de référence de l'énoncé $(\mathrm{t})$ et le moment de l'énonciation $\left(\mathrm{t}_{0}\right)$, elle introduit un «moment de la présupposition » $\mathrm{t}_{\mathrm{p}}$ (le moment antérieur à l'énonciation que le locuteur construit en «présupposant ») et un «moment présupposé » t'. Dans le modèle, seules deux relations sont prédéfinies : (i) la relation entre $t$ et $\mathrm{t}^{\prime}$ ( $\mathrm{t}^{\prime}$ est immédiatement postérieur à $\mathrm{t}$ ) et (ii) la relation entre $\mathrm{t}_{\mathrm{p}}$ et $\mathrm{t}_{0}\left(\mathrm{t}_{\mathrm{p}}\right.$ est immédiatement ${ }^{15}$ antérieur à $\left.\mathrm{t}_{0}\right)$. La relation entre $\mathrm{t}-\mathrm{t}^{\prime}$ d'une part et $\mathrm{t}_{\mathrm{p}}-\mathrm{t}_{0}$ d'autre part est quant à elle fonction de la localisation de $t$ par rapport à $t_{0}$, c'est-à-dire du temps grammatical de Déjà $P(t)$ :

15 En introduisant l'adverbe «immédiatement» dans ces définitions, nous ne cherchons pas à introduire une quelconque proximité entre les moments concernés mais à établir que les paires ainsi formées ( $\mathrm{t}$ et $\mathrm{t}^{\prime}$ d'une part et $\mathrm{t}_{\mathrm{p}}$ et $\mathrm{t}_{0}$ d'autre part) sont indivisibles. Cette distinction entre contiguïté et immédiateté est possible dans la mesure où la représentation du temps sur laquelle s'appuie l'hypothèse présentée ne comporte que quatre moments (et non une succession infinie d'instants contigus comme la représentation traditionnelle). 
Version pre-print. A paraître dans Langages.

- Si Déjà $P(t)$ est au passé, alors t et $\mathrm{t}^{\prime}$ sont antérieurs à $\mathrm{t}_{\mathrm{p}}$ et $\mathrm{t}_{0}$. On a : $\mathrm{t}<\mathrm{t}^{\prime}<\mathrm{t}_{\mathrm{p}}<\mathrm{t}_{0}$.

- Si Déjà $P(t)$ est au présent, alors $\mathrm{t}$ et $\mathrm{t}_{0}$ coïncident. On $\mathrm{a}: \mathrm{t}_{\mathrm{p}}<\mathrm{t}=\mathrm{t}_{0}<\mathrm{t}^{\prime}$.

Ainsi formulé, le modèle 3 prévoit que l'adverbe déjà introduit une vision prospective dans les énoncés au présent et une vision rétrospective dans les énoncés au passé :

- Dans les énoncés au présent, $\mathrm{t}$ ' est postérieur à $\mathrm{t}_{\mathrm{p}}$. «On savait en $\mathrm{t}_{\mathrm{p}}$ que $\mathrm{P}$ être-vrai en $\mathrm{t}$ ' » se lit donc : «On savait en $t_{p}$ que P serait vrai en $t$ ' ».

- Dans les énoncés au passé, $t$ ' est antérieur à $t_{p}$. «On savait en $t_{p}$ que $\mathrm{P}$ être-vrai en t' » se lit donc :

«On savait en $t_{p}$ que $P$ était vrai en $t$ ' ».

Selon nous, c'est cette différence entre les énoncés au présent et les énoncés au passé qui permet d'expliquer pourquoi déjà marque la réalisation d'une expectative en (2) et une continuation en (3) :

Application du modèle 3 aux énoncés (2) et (3)

(2) Paul veut déjà être ministre.

Les contenus (2b) et (2a) correspondent respectivement au présupposé et au posé de (2) :

Contenu (2b) : En énonçant (2), le locuteur fait comme si on savait, avant l'énonciation, que [Paul vouloir être ministre] serait vrai à un moment postérieur au moment de l'énonciation. Autrement dit, on s'attendait à ce que Paul ressente un jour l'envie d'être ministre.

Contenu (2a): Dans ce cadre, le locuteur asserte que [Paul vouloir être ministre] est vrai au moment de l'énonciation. Autrement dit, [Paul vouloir être ministre] ne sera pas seulement vrai plus tard, c'est vrai aussi maintenant. Ce que l'on prévoyait s'est réalisé.

Contenu (2c) : Selon la loi d'informativité de Ducrot (1972), «tout énoncé $A$, s'il est présenté comme source d'information, induit le sous-entendu que le destinataire ignore $A$ ou même, éventuellement, qu'on s'attendrait plutôt à non- $A \gg$ (Ducrot, 1972, 133). En (2), le locuteur sous-entend que l'on aurait pu s'attendre à ce que [Paul vouloir être ministre] ne soit pas vrai maintenant mais seulement plus tard. [Paul vouloir être ministre] est donc vrai plus tôt que prévu.

(3) En 2007, Paul voulait déjà être ministre.

Les contenus (3b) et (3a) correspondent respectivement au présupposé et au posé de (3) :

Contenu (3b) : En énonçant (3), le locuteur fait comme si on savait, avant l'énonciation, que [Paul vouloir être ministre] était vrai à un moment postérieur à 2007.

Contenu (3a) : Dans ce cadre, il asserte que [Paul vouloir être ministre] était vrai en 2007. Autrement dit, [Paul vouloir être ministre] n'était pas seulement vrai après 2007, c'était vrai aussi en 2007. La phase postérieure à 2007 dont on avait connaissance n'est pas le début du procès [Paul vouloir être ministre] mais seulement une continuation.

On remarquera que l'application du modèle 3 que nous proposons fait la part belle à l'antériorité temporelle du présupposé. Selon nous, le moment où l'on « se représente » le présupposé n'est ni le moment de référence de l'énoncé ${ }^{16}$, ni le moment de l'énonciation ${ }^{17}$. Le moment où l'on se représente le présupposé est un moment antérieur au moment de l'énonciation, construit par la présupposition. Si le présupposé véhiculé par déjà est interprété comme une expectative et non comme une continuation dans les énoncés au présent, c'est parce que le moment futur qu'il évoque n'est pas seulement futur par rapport au moment de l'énonciation, mais aussi et surtout par rapport à un moment antérieur au moment de l'énonciation. De même, si la continuation marquée par déjà dans les énoncés au passé est présentée d'une manière peu habituelle (la phase postérieure étant envisagée avant la phase antérieure), c'est parce que le présupposé se conçoit dans l'antériorité du posé.

\section{En guise de conclusion}

\footnotetext{
${ }^{16}$ En insistant sur ce point, nous nous opposons par exemple à Nef (1986: 257) pour qui dire que Encore $P(t)$ présuppose qu'il est possible que $\mathrm{P}$ ne soit pas vrai après $\mathrm{t}$ conduit à dire que l'énoncé Paul, bien que nonagénaire, était encore capable de chasser le sanglier présuppose qu' " il a été le cas qu'il sera le cas qu'il est possible que non $\mathrm{p}$ ».

${ }^{17}$ En insistant sur ce point, nous nous opposons cette fois à Martin (1983b : 38) qui, à plusieurs reprises dans son ouvrage, associe les présuppositions à «l'univers de locuteur». Or, pour Martin, l'univers du locuteur est l'ensemble des propositions que le locuteur tient pour vraies au moment où il s'exprime.
} 
Version pre-print. A paraître dans Langages.

L'étude d'une construction présuppositionnelle non paradigmatique telle que l'adverbe déjà éclaire d'un jour nouveau certaines questions concernant la présupposition. D'une part, elle permet de montrer que les divergences entre les approches vériconditionnelle et sémantico-pragmatique de la notion sont beaucoup plus importantes qu'il n'y paraît. D'autre part, elle conduit à mettre en avant certaines propriétés de la présupposition habituellement peu exploitées, telles que l'antériorité temporelle du présupposé. ${ }^{18}$

\section{Références bibliographiques}

ABRAHAM W. (1980), "The synchronic and diachronic semantics of German temporal noch and schon, with aspects of english still, yet and already", Studies in Language 4, 3-24.

ANSCOMBRE J.C. (2005), " Le ON-locuteur, une entité aux multiples visages », in J. Bres, P.P. Haillet, S. Mellet, H. Nølke \& L. Rosier (éds), Dialogisme, polyphonie : approches linguistiques, Louvain-la-Neuve : Duculot, 75-94.

DELOOR S. (2006), Pour un traitement sémantique et pragmatique de la particule ya en espagnol contemporain, Paris : Ecole des Hautes Etudes en Sciences Sociales, Thèse de doctorat.

DEloor S. (2010), "J'ai déjà mangé: expérience ou résultat?», Revue de sémantique et pragmatique 28, 25-46.

DOHERTY M. (1973), "Noch and schon and their presuppositions", in F. Kiefer \& N. Ruwet (éds.), Generative Grammar in Europe, Dordrecht : Reidel, 154-177.

GARRIDO MEDINA J. (1991), « Adverbs and particles of change and continuation : Spanish todavía and $y a »$, EUROTYP Working Papers, 5.2., pp. 43-58.

GARRIDO MEDINA J. (1992), "Expectations in Spanish an German adverbs of change", Folia lingüística $26: 3-4,357-402$.

GIRÓN ALCONCHELL J.L. (1991) Tiempo, modalidad y adverbio : significado y función de ya, Universidad de Salamanca, Salamanca.

Horn L.R. (1970), “Ain’t it Hard (Anymore)”, in M.A. Campbell \& alii (éds.), Papers from the Sixth Regional Meeting of Chicago Linguistic Society, Chicago, 318-327.

HORN L. (1997), "Presupposition and implicature", in S. Lappin (ed.), The Handbook of Contemporary Semantic Theory, Oxford : Blackwell's, 1995, pp. 299-319.

KÖNIG E. (1977), “Temporal and non-temporal uses of noch and schon in German”, Linguistics and Philosophy 1.2, 167-212.

LÖBNER S. (1989), "German schon - erst - noch : an integrated analysis", Linguistics and Philosophy 12, 167-212.

LÖBNER S. (1999), "Why German schon and noch are still duals", Linguistics and Philosophy 22, 45-107.

Martin R. (1983), Pour une logique du sens, Paris : PUF.

Michaelis L. (1996), «On the use and meaning of already », Linguistics and Philosophy, 19, pp. 477-502.

MiтTwoch A. (1993), «The relationship between schon / already and noch / still : a reply to Löbner », Natural language semantics, 2, pp. 71-82.

MULLER C., (1975), «Remarques syntactico - sémantiques sur certains adverbes de temps », Le français moderne 43, 12-38.

NEF F. (1986), Sémantique de la référence temporelle en français moderne, Peter Lang, Bern.

VAN DER AUWERA J. (1993), «Already and still : Beyond Duality », Linguistics and Philosophy, 16, pp. 613-653.

\footnotetext{
${ }^{18}$ Je remercie pour son aide J.C. Anscombre, avec qui j'ai eu de nombreuses discussions sur plusieurs des points abordés dans cet article.
} 